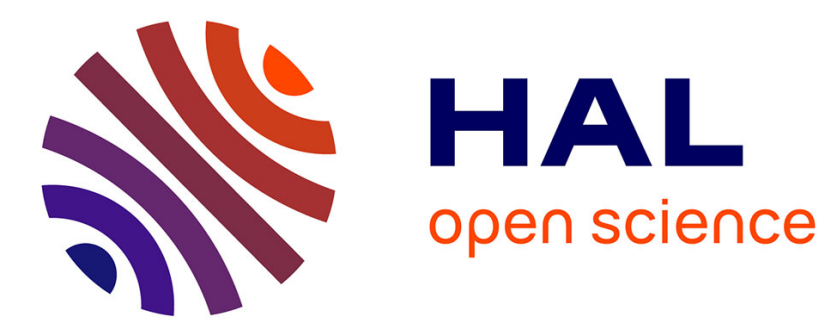

\title{
Experimental identification of an uncertain computational dynamical model representing a family of structures
}

Anas Batou, Christian Soize, M. Corus

\section{- To cite this version:}

Anas Batou, Christian Soize, M. Corus. Experimental identification of an uncertain computational dynamical model representing a family of structures. Computers \& Structures, 2011, 89 (13-14), pp.1440-1448. 10.1016/j.compstruc.2011.03.004 . hal-00684292

\section{HAL Id: hal-00684292 \\ https://hal.science/hal-00684292}

Submitted on 31 Mar 2012

HAL is a multi-disciplinary open access archive for the deposit and dissemination of scientific research documents, whether they are published or not. The documents may come from teaching and research institutions in France or abroad, or from public or private research centers.
L'archive ouverte pluridisciplinaire HAL, est destinée au dépôt et à la diffusion de documents scientifiques de niveau recherche, publiés ou non, émanant des établissements d'enseignement et de recherche français ou étrangers, des laboratoires publics ou privés. 


\title{
Experimental identification of an uncertain computational dynamical model representing a family of structures
}

\author{
A.Batou*a, C.Soize ${ }^{\mathrm{a}}$, M.Corus $^{\mathrm{b}}$ \\ ${ }^{a}$ Université Paris-Est, Laboratoire Modélisation et Simulation Multi Echelle, MSME \\ UMR 8208 CNRS, 5 bd Descartes, 77454 Marne-la-Vallee, France \\ ${ }^{b}$ LaMSID, CNRS-EDF, UMR 2832, 1 avenue du général De Gaulle, 92140 Clamart, \\ France
}

\begin{abstract}
We are interested in constructing an uncertain computational model representing a family of structures and in identifying this model using a small number of experimental measurements of the first eigenfrequencies. The prior probability model of uncertainties is constructed using the generalized probabilistic approach of uncertainties which allows both system-parameters uncertainties and model uncertainties to be taken into account. The parameters of the prior probability model of uncertainties are separately identified for each type of uncertainties, yielding an optimal prior probability model. The optimal prior stochastic computational model allows a robust analysis for the family of structures to be carried out.
\end{abstract}

Keywords:

\section{Introduction}

To ensure the security of its installations, EDF (Electricité De France) regularly and frequently performs a large variety of experimental tests on the various components of the electric power plants. Among these components, the Containment Spray System (CSS) motors are structures made up of a

A. Batou, C. Soize, M. Corus, Experimental identification of an uncertain computational dynamical model representing a family of structures, Computers and Structures, 89(13-14), 1440-1448, 2011. 
motor, a holding system (junction), a housing and others structural elements (described in Fig. 1). A CSS motor is used in case of emergency. It drives the pump used to spray coolant into the primary containment structure, and avoids overpressure. Sometimes, vibration of CSS motors can reach the alarm level. These vibrations mainly occur in the neighborhood of the rotation frequency, where the first three eigenmodes of a CSS motor are located. We are then interested in the range of variation of the first three eigenfrequencies of a CSS motor belonging to a family of motors. The rotation frequency of such a motor is fixed. In this paper, all experimental and numerical frequencies have been divided by this given rotation frequency and are therefore dimensionless. Consequently, the dimensionless rotation frequency is equal to 1 . The target is such that the first three dimensionless eigenfrequencies have to be out of the range $[0.92,1.08]$ in order to avoid a resonance induced by the rotation of motors. For a given motor belonging to the family, the first eigenfrequency is generally lower than the rotation frequency, and corresponds to a rocking motion of the motor, coupled with the first flexural mode of its concrete slab, in phase with the bending of the housing. The second eigenfrequency, close to the rotation frequency, involves quite the same shape, in an orthogonal direction, but without coupling with the concrete slab. The third eigenfrequency still exhibits a flexural motion of the housing in phase with the rocking of the motor, but with an out of phase coupling with the first flexural mode of the concrete slab. Many solutions have been tested to solve vibration issues, either in stiffening the assembly (stiffeners clamped to the surrounding walls, modifications of the junction between the motor and the floor, new piles to support the floor, etc.), or in lowering its eigenfrequencies (using added masses). However, due to the large dispersion exhibited by the eigenfrequencies of these structures, no generic solution could be found.

To overcome this issue, to better understand the behavior of these motors, and to help designing a general solution, an unique uncertain computational dynamical model representing the considered family of structures must be built.

The dynamical behavior of a nominal CSS motor is described using a computational dynamical model for which there are two types of uncertainties. The first one, referred as system-parameters uncertainties, is related to some parameters of the computational dynamical model which are considered as uncertain. The second one, referred as model uncertainties, is due to the modeling errors which are due to the simplifications with respect to the real motors, introduced in the computational dynamical model (see Sec- 
tion 3). One objective is to model uncertainties in the computational model using a probabilistic approach. The construction of such a stochastic computational model is usually performed in two steps. The first one consists in constructing a prior probability model of the random variables which models uncertain quantities of the computational model. If experimental data are available, the second one consists in constructing a posterior probability model derived from the prior probability model and from the experimental data, using the Bayesian method [2]. If there are a lot of experimental data, the prior probability model can be rough enough, because the Bayesian approach allows a good probability model to be fitted. If there are no experimental data, the posterior model can not be constructed. If only few experimental data are available, and if the prior probability model is too rough, then the posterior model will stay relatively rough. In these two last cases, it is necessary to construct the best prior probability model using the available information. A method consists in constructing the prior probability distribution using the maximum entropy principle under the constraints defined by the available information. We then obtain an algebraic representation of the probability distribution which depends on unknown parameters. If there are no experimental data, then these unknown parameters must be considered as parameters to perform a sensitivity analysis. If there are few experimental data, these unknown parameters can be estimated using data and the maximum likelihood method. In this paper, we are in this last situation. There are only a few experimental data. We then construct a prior probability model depending on unknown parameters which are estimated with maximum likelihood method. Such a prior probability model for which its unknown parameters are estimated with experimental data will be called below the optimal prior probability model.

Concerning system-parameter uncertainties, the main method is based on the use of the parametric probabilistic approach which has extensively been developed in the last three decades, which is still in development and which allows the uncertain system parameters of the nominal model to be taken into account through the introduction of a prior probability model of these system parameters (see for instance [5], [15], [19], [16], [20], [21]). Such an approach consists (1) either in constructing an adapted representation based on a polynomial chaos decomposition (see for instance [5], [8], [7], [14], [24], [6], [19], [4], [3], [9], [1],[28], and see the recent synthesis presented in [13]), (2) or in directly constructing the probability distribution of the random quantity using the available information and the Maximum Entropy Principle 
introduced by Jaynes [10] in the context of Information Theory developed by Shannon [23] (see for instance [12], [11] and for a recent development in this field $[26])$.

Concerning model uncertainties induced by modeling errors, it is today well understood that prior and posterior probabilistic models of the uncertain model parameter are not sufficient and do not have the capability to take into account model uncertainties as explained in the context of computational mechanics (see for instance [2], [25]). Two main methods can be used to take into account model uncertainties (modeling errors). (1) The first one consists in introducing a probabilistic model of the output prediction error which is the difference between the real system output and the model output (note that such a probabilistic approach of model uncertainties is implemented at the output level of the nominal model and not implemented at the operator level of the model). A posterior probabilistic model can be constructed using, for instance, the Bayesian approach (see for instance [2]). (2) The second one is based on the nonparametric probabilistic approach of model uncertainties (modeling errors) which is described in [25] as another possible way to the use of the output-prediction-error method in order to take into account modeling errors. The nonparametric probabilistic approach consists in directly constructing the stochastic modeling of the operators of the nominal computational model instead of introducing a probabilistic model of the prediction errors.

Since there are two types of uncertainties (system-parameters uncertainties and model uncertainties) and since the two types must be separately identified using experimental data, we propose to use the generalized probabilistic approach of uncertainties which has recently been proposed (see [27]) to construct a prior probabilistic model of uncertainties. This method allows both system-parameters uncertainties and model uncertainties to be taken into account. In this method, the model errors are taken into account using the nonparametric probabilistic approach. The prior probabilistic model of each type of uncertainties is constructed and identified in a separate way using the maximum likelihood method with the experimental data, yielding an optimal prior probability model. Such a methodology is applied (1) to the construction of a unique prior stochastic computational dynamical model representing all the CSS motors belonging to the considered family and (2) to the identification of this prior stochastic computational model with experimental measurements yielding an optimal prior stochastic computational model. Finally, the optimal prior stochastic computational model allows statistics 
on the first three eigenfrequencies to be constructed and allows a robust analysis for the family of structures to be carried out. This optimal prior stochastic computational model could be improved in constructing a posterior stochastic computational model consisting in reusing the experimental data and the Bayesian method in order to construct a posterior probability model of uncertainties. Nevertheless, we will see that there are 8 independent random variables to model uncertain system parameters, and 2 random matrices which are positive-definite symmetric $4 \times 4$ real matrices for which the random entries are mutually dependent. As it will be shown, each random matrix will be a nonlinear transformation of 10 independent random germs. Consequently, the stochastic modeling of uncertainties will introduce 28 independent random variables. Since only 11 experimental data are available, it can be thought that such a posterior model of a probability distribution on a subset of $\mathbb{R}^{28}$ cannot reasonably be improved with respect to the optimal prior model using only 11 experimental data. This is the reason why a posterior model has not be constructed. Finally, it should be noted that such the presentation of the construction of an optimal prior model using the generalized approach and experimental data has never been previously carried out.

Section 2 is devoted to the description of a CSS motor and to the experimental measurements performed on the family of CSS motors. Sections 3 and 4 present the unique nominal computational dynamical model and the unique stochastic computational dynamical model. Section 5 deals with the experimental identification of the parameters of the prior probability model of uncertainties yielding an optimal prior probability model. In the last Section, statistics on the three first eigenfrequencies are constructed using the optimal prior stochastic computational dynamical model. Such statistics allow a robust analysis to be carried out for the family of CSS motors.

\section{Dynamical system under consideration and its related experi- mental measurements}

We are interested in the prediction (in a statistical sense) of the first three eigenfrequencies of a family of CSS motors represented by a unique computational dynamical model for which there are both system-parameters uncertainties and model uncertainties. A CSS motor is linked to a flexible base plate which is bolted on a steel plate. This metallic plate is bonded to an upper flexible concrete floor, and connected to a pump installed on a 
lower floor using a driving shaft. This driving shaft is protected by a housing clamped to the base plate, as shown in Fig. 1. The motor is a very rigid

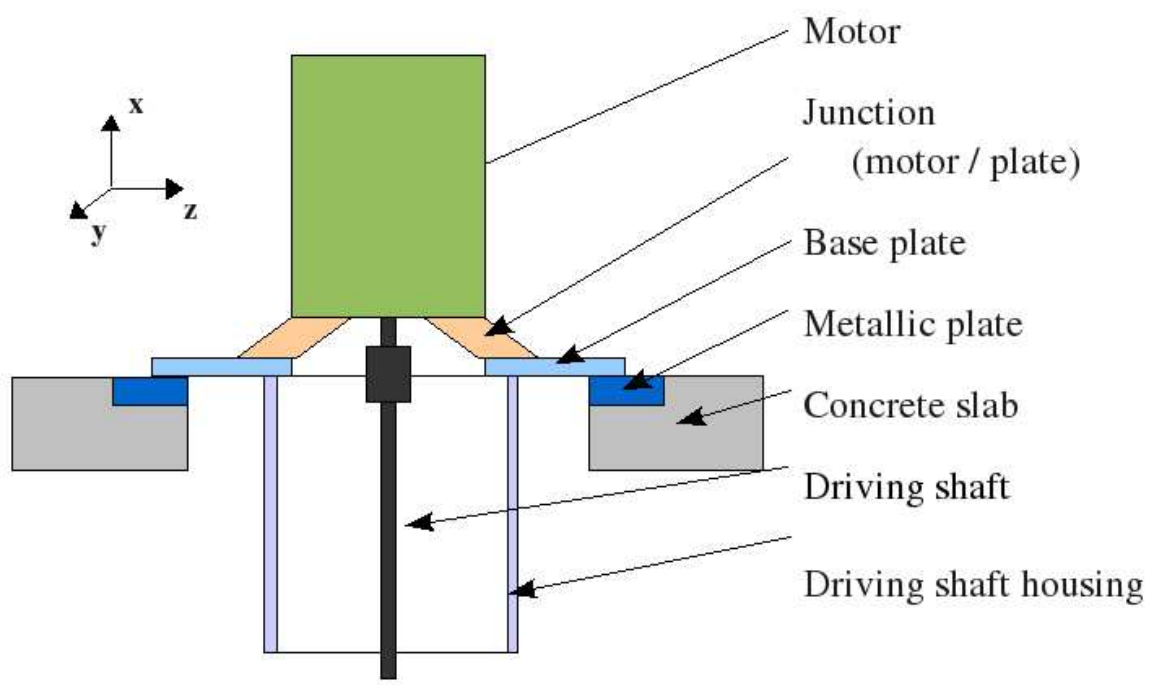

Figure 1: Scheme defining a CSS motor

assembly, while the housing is flexible. The considered family is constituted of 11 CSS motors. Each motor belonging to this family is experimentally tested. The first eigenfrequencies are experimentally identified. It should be noted that the measured frequency response functions are not available. For the 11 CSS motors of the considered family, the first eigenfrequencies which are measured are given (in dimensionless form) in Table 1.

\begin{tabular}{|c|c|c|c|c|c|c|c|c|c|c|c|}
\hline$i$ & 1 & 2 & 3 & 4 & 5 & 6 & 7 & 8 & 9 & 10 & 11 \\
\hline$f_{1}^{\text {exp }, i}$ & 0.77 & 0.78 & 0.77 & 0.81 & 0.85 & 0.97 & 0.85 & 0.91 & 0.96 & 0.85 & 0.95 \\
\hline$f_{2}^{\text {exp }, i}$ & 0.84 & 0.86 & 0.79 & 0.85 & 0.90 & 0.98 & 0.92 & 0.92 & 1.04 & 0.88 & 0.98 \\
\hline$f_{3}^{\text {exp }, i}$ & 1.01 & 1.13 & 0.85 & 1.02 & 1.0 & - & - & - & - & - & - \\
\hline
\end{tabular}

Table 1: Measured eignefrequencies.

It should be noted that the third eigenfrequency is measured only for 5 CSS motors. In addition, Table 1 shows a large dispersion for the first 
three eigenfrequencies with an overlapping between the eigenfrequencies of the CSS motors.

\section{Nominal computational dynamical model}

The nominal computational dynamical model is the deterministic designed computational model which is constructed to represent all the CSS motors belonging to the considered family.

The mesh of the finite element model of this nominal computational dynamical model is represented in Fig. 2. The concrete slab, the base plate and the metallic plate are modeled by volume finite elements. The housing is modeled by a Timoshenko beam for which the area of the section is $A=5.4 \times 10^{-3} \mathrm{~m}^{2}$ and the bending inertia coefficients are $I_{y}=I_{z}=$ $3.36 \times 10^{-5} \mathrm{~m}^{4}$. The motor is modeled by a rigid body for which the total mass, the position of the center of mass and the tensor of inertia reduced at the center of mass are defined. The junction between the motor and the base plate is modeled by a rotation spring for which the rotation stiffness with respect to the two directions of bending of the structure are both equal to $k_{r}=6.8 \times 10^{7} \mathrm{Nm} / \mathrm{rad}$. The characteristics of the materials are summarized in Tab. 2. The four lateral sides of the concrete slab are fixed. The finite element model has 40,866 DOF. The eigenfrequencies $f_{1}, f_{2}, \ldots$ are calcu-

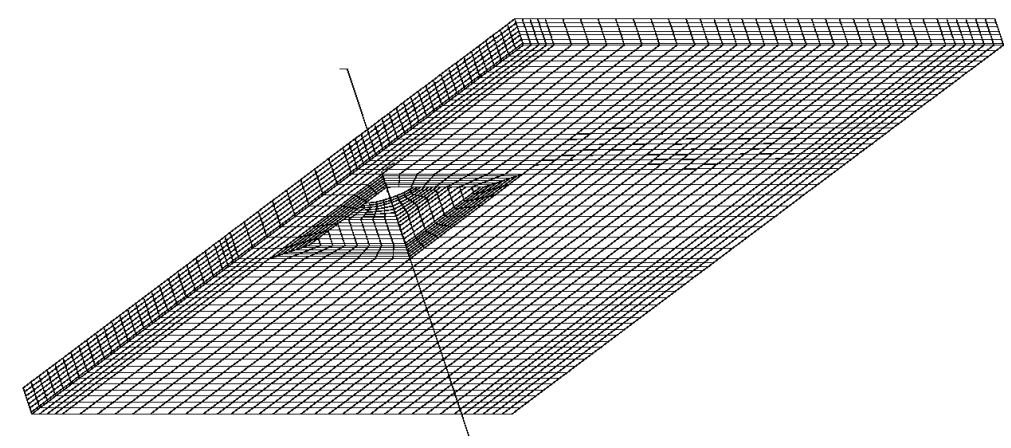

Figure 2: FE model

lated by finding the solutions $\omega=2 \pi f$ and $\boldsymbol{\varphi}$ of the generalized eigenvalue problem,

$$
[K] \boldsymbol{\varphi}=\omega^{2}[M] \varphi
$$




\begin{tabular}{|c|c|c|c|}
\hline & Mass density & Young modulus & Poisson modulus \\
\hline Concrete slab & $2.4 \times 10^{3} \mathrm{~kg} / \mathrm{m}^{3}$ & $1.25 \times 10^{10} \mathrm{~N} / \mathrm{mm}^{2}$ & 0.17 \\
\hline Base plate & $7.8 \times 10^{3} \mathrm{~kg} / \mathrm{m}^{3}$ & $2.1 \times 10^{11} \mathrm{~N} / \mathrm{mm}^{2}$ & 0.3 \\
\hline Metallic plate & $7.8 \times 10^{3} \mathrm{~kg} / \mathrm{m}^{3}$ & $2.1 \times 10^{11} \mathrm{~N} / \mathrm{mm}^{2}$ & 0.3 \\
\hline Gimbal housing & $7.8 \times 10^{3} \mathrm{~kg} / \mathrm{m}^{3}$ & $2.1 \times 10^{11} \mathrm{~N} / \mathrm{mm}^{2}$ & 0.3 \\
\hline
\end{tabular}

Table 2: Caracteristics of the materials.

in which $[M]$ and $[K]$ are respectively the positive-definite symmetric real mass and stiffness matrices (there is no rigid body mode). Let $[\Phi]=\left[\boldsymbol{\varphi}_{1} \ldots \boldsymbol{\varphi}_{n}\right]$ be the matrix of the $n$ eigenmodes associated with the $n$ eigenfrequencies ordered by increasing values $\left(f_{1} \leq f_{2} \leq \ldots \leq f_{n}\right)$. Then the generalized mass and stiffness matrices are defined by $[\widetilde{M}]=[\Phi]^{T}[M][\Phi]$ and $[\widetilde{K}]=[\Phi]^{T}[K][\Phi]$. The three eigenmodes associated with the first three eigenfrequencies $\left(f_{1}=0.82, f_{2}=0.87, f_{3}=1.11\right)$ which are computed with the nominal computational dynamical model, are shown in Figs. 3, 4 and 5.

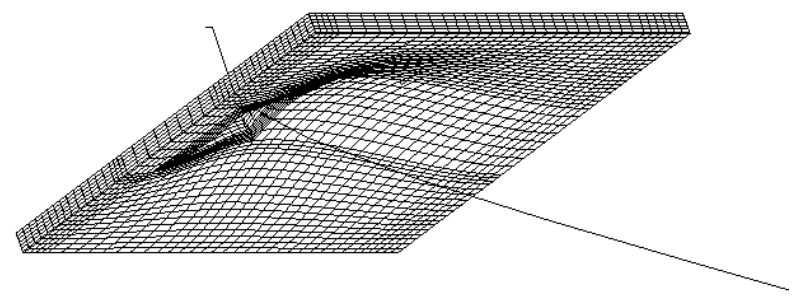

Figure 3: First eigenmode calculated with the nominal computational dynamical model

\section{Stochastic computational dynamical model}

As explained in Section 2, the nominal computational model presented in Section 3 owns two types of uncertainties: system-parameters uncertainties and model uncertainties induced by the modeling errors. The systemparameters uncertainties are mainly induced by the variability of the eight parameters $x_{1}, \ldots, x_{8}$ of the nominal computational model: (1) the mass density $\rho_{c s}$, the two Lame coefficients $\lambda_{c s}$ and $\mu_{c s}$ of the concrete slab, (2) 


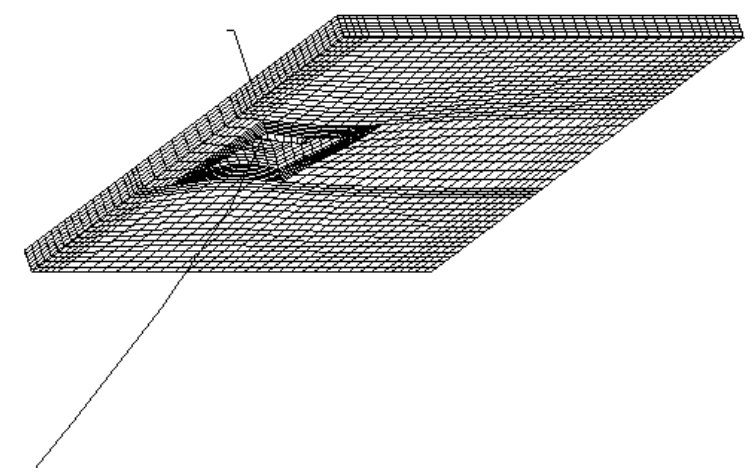

Figure 4: Second eigenmode calculated with the nominal computational dynamical model

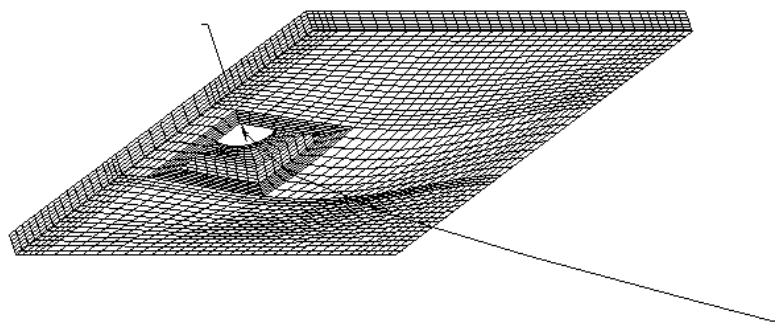

Figure 5: Third eigenmode calculated with the nominal computational dynamical model

the mass density $\rho_{m p}$, the two Lame coefficients $\lambda_{m p}$ and $\mu_{m p}$ of the metallic plate, (3) the bending inertia coefficient $I_{h}$ of the housing and the rotation stiffness $k_{s}$ of the spring. Let $\boldsymbol{x}=\left(x_{1}, \ldots, x_{8}\right)$ be the vector representing all the uncertain system parameters $\left(\rho_{c s}, \lambda_{c s}, \mu_{c s}, \rho_{m p}, \lambda_{m p}, \mu_{m p}, I_{h}, k_{s}\right)$. Vector $\boldsymbol{x}$ belongs to the admissible set denoted by $\mathcal{C}_{x}$.

The two types of uncertainties are taken into account using the generalized probabilistic approach of uncertainties introduced in [27] and which allows an independent modeling of both system-parameters uncertainties and modeling errors to be separately taken into account and experimentally identified.

4.1. Construction of the probability model of the uncertain system-parameters

Let $E\{$.$\} be the mathematical expectation. The eight uncertain parame-$ ters $\left(x_{1}, \ldots, x_{8}\right)$ are modeled by the random vector $\boldsymbol{X}=\left(X_{1}, \ldots, X_{8}\right)$ defined 
on the probability space $(\Theta, \mathcal{T}, \mathcal{P})$. The probability distribution of random vector $\boldsymbol{X}$ which models the uncertain system-parameters is constructed using the Maximum Entropy Principle (MaxEnt) from Information Theory [23], under the constraints defined by the available information [10]. Since, no information is available concerning the statistical correlation between the components $X_{1}, \ldots, X_{8}$ of $\boldsymbol{X}$, the use of the MaxEnt principle yields, as a result, that the real-valued random variables $X_{1}, \ldots, X_{8}$ are independent. Consequently, for any fixed $i$, the probability density function of random variable $X_{i}$ is constructed using the MaxEnt principle under the constraints defined by the following available information: (1) Random variable $X_{i}$ must be positive almost surely. (2) The mean value of random variable $X_{i}$ must be equal to the value $\underline{x}_{i}$ of the nominal computational model. (3) Random variable $X_{i}$ must verify the inequality $E\left\{X_{i}^{-2}\right\}<+\infty$ in order that the random eigenvalues be second-order random variables. It can be proven that the third constraint can be replaced by $\left|E\left\{\log X_{i}\right\}\right|<+\infty$, this last one implying the previous one. It can then be deduced that the probability density function $p_{X_{i}}\left(x_{i}\right)$ with respect to $d x_{i}$ of random variable $X_{i}$ is written as

$p_{X_{i}}\left(x_{i} ; \delta_{x_{i}}\right)=\mathbb{1}_{] 0,+\infty[}\left(x_{i}\right) \frac{1}{\underline{x}_{i}}\left(\frac{1}{\delta_{x_{i}}^{2}}\right)^{\frac{1}{\delta_{x_{i}}^{2}}} \frac{1}{\Gamma\left(1 / \delta_{x_{i}}^{2}\right)}\left(\frac{x_{i}}{\underline{x}_{i}}\right)^{\frac{1}{\delta_{x_{i}}^{2}}-1} \exp \left(-\frac{x_{i}}{\delta_{x_{i}}^{2} \underline{x}_{i}}\right)$,

where $\Gamma(\alpha)=\int_{0}^{+\infty} t^{\alpha-1} e^{-t} d t$ is the Gamma function and where $\mathbb{1}_{0,+\infty[}(x)=$ 1 if $x>0$ and $=0$ if not. The dispersion parameter $\delta_{x_{i}}$ is defined as the coefficient of variation given by $\delta_{x_{i}}=\sigma_{X_{i}} / \underline{x}_{i}$ where $\sigma_{X_{i}}$ is the standard deviation of random variable $X_{i}$, such that $0 \leq \delta_{x_{i}}<1 / \sqrt{2}$. Therefore, the probability density function of the random variable $X_{i}$ is completely defined by the mean value $\underline{x}_{i}$ which is given by the nominal computational model and by the dispersion parameter $\delta_{x_{i}}$ which must be identified using the measured eigenfrequencies. Let $F_{1} \leq \ldots \leq F_{n}$ the order statistics of the real-valued random variables $F_{1}, \ldots, F_{n}$ modeling the eigenfrequencies $f_{1} \leq \ldots \leq f_{n}$. For any realization $\boldsymbol{X}(\theta)$ of the random variable $\boldsymbol{X}$ with $\theta$ in $\Theta$, the realizations $F_{1}(\theta) \leq \ldots \leq F_{n}(\theta)$ of the random eigenfrequencies $F_{1}, \ldots, F_{n}$ are calculated by finding the solutions $\omega(\boldsymbol{X}(\theta))=2 \pi f(\boldsymbol{X}(\theta))$ and $\boldsymbol{\varphi}(\boldsymbol{X}(\theta))$ of the following random generalized eigenvalue problem,

$$
[K(\boldsymbol{X}(\theta))] \boldsymbol{\varphi}(\boldsymbol{X}(\theta))=\omega(\boldsymbol{X}(\theta))^{2}[M(\boldsymbol{X}(\theta))] \boldsymbol{\varphi}(\boldsymbol{X}(\theta)) .
$$


This random generalized eigenvalue problem is solved using the Monte Carlo Simulation Method (note that there exist efficient algorithms to solve such a stochastic generalized eigenvalue problem for large systems such as [17]). The $(n \times n)$ random generalized mass and stiffness matrices are then defined by

$$
\begin{aligned}
& {[\widetilde{M}(\boldsymbol{X}(\theta))]=[\Phi(\boldsymbol{X}(\theta))]^{T}[M(\boldsymbol{X}(\theta))][\Phi(\boldsymbol{X}(\theta))]} \\
& {[\widetilde{K}(\boldsymbol{X}(\theta))]=[\Phi(\boldsymbol{X}(\theta))]^{T}[K(\boldsymbol{X}(\theta))][\Phi(\boldsymbol{X}(\theta))] .}
\end{aligned}
$$

Since random matrices $[\widetilde{M}(\boldsymbol{X}(\theta))]$ and $[\widetilde{K}(\boldsymbol{X}(\theta))]$ are positive definite, their Cholesky decomposition yields

$$
\begin{aligned}
& [\widetilde{M}(\boldsymbol{X}(\theta)))]=\left[L_{M}(\boldsymbol{X}(\theta))\right]^{T}\left[L_{M}(\boldsymbol{X}(\theta))\right], \\
& \left.[\widetilde{K}(\boldsymbol{X}(\theta))]=\left[L_{K}(\boldsymbol{X}(\theta))\right)\right]^{T}\left[L_{K}(\boldsymbol{X}(\theta))\right] .
\end{aligned}
$$

\subsection{Construction of the probability model of model uncertainties}

The construction of the probability model of model uncertainties is carried out as explained in [27]. Therefore, the dependent random matrices $[\widetilde{M}(\boldsymbol{X})]$ and $[\widetilde{K}(\boldsymbol{X})]$ are replaced by the dependent random matrices $[\widetilde{\boldsymbol{M}}(\boldsymbol{X})]$ and $[\widetilde{\boldsymbol{K}}(\boldsymbol{X})]$. For any fixed value of vector $\boldsymbol{x}$ in admissible set $\mathcal{C}_{\boldsymbol{x}}$, the probability density function of the random matrices $[\widetilde{\boldsymbol{M}}(\boldsymbol{x})]$ and $[\widetilde{\boldsymbol{K}}(\boldsymbol{x})]$ are constructed using the MaxEnt principle under the constraints defined by the following available information: (1) the random matrices $[\widetilde{\boldsymbol{M}}(\boldsymbol{x})]$ and $[\widetilde{\boldsymbol{K}}(\boldsymbol{x})]$ must be positive definite almost surely. (2) The mean value of the random matrices $[\widetilde{\boldsymbol{M}}(\boldsymbol{x})]$ and $[\widetilde{\boldsymbol{K}}(\boldsymbol{x})]$ are respectively equal to $[\widetilde{M}(\boldsymbol{x})]$ and $[\widetilde{K}(\boldsymbol{x})]$. (3) The random matrices $[\widetilde{\boldsymbol{M}}(\boldsymbol{x})]$ and $[\widetilde{\boldsymbol{K}}(\boldsymbol{x})]$ must verify the inequalities $E\left\{\left\|[\widetilde{\boldsymbol{M}}(\boldsymbol{x})]^{-1}\right\|^{2}\right\}<+\infty$ and $E\left\{\left\|[\widetilde{\boldsymbol{K}}(\boldsymbol{x})]^{-1}\right\|^{2}\right\}<+\infty$ in order that the random eigenvalues of the stochastic computational dynamical model be second-order random variables. In addition, it can be proven that the third constraint can be replaced by the following one: $|E\{\log (\operatorname{det}[\widetilde{\boldsymbol{M}}(\boldsymbol{x})])\}|<+\infty$ and $|E\{\log (\operatorname{det}[\widetilde{\boldsymbol{K}}(\boldsymbol{x})])\}|<+\infty$. Taking into account Eqs. (6) and (7), the random matrices $[\widetilde{\boldsymbol{M}}(\boldsymbol{x})]$ and $[\widetilde{\boldsymbol{K}}(\boldsymbol{x})]$ are written as,

$$
\begin{aligned}
{[\widetilde{\boldsymbol{M}}(\boldsymbol{x})] } & =\left[L_{M}(\boldsymbol{x})\right]^{T}\left[\mathbf{G}_{M}\right]\left[L_{M}(\boldsymbol{x})\right] \\
{[\widetilde{\boldsymbol{K}}(\boldsymbol{x})] } & =\left[L_{K}(\boldsymbol{x})\right]^{T}\left[\mathbf{G}_{K}\right]\left[L_{K}(\boldsymbol{x})\right] .
\end{aligned}
$$


in which the random matrices $\left[\mathbf{G}_{M}\right]$ and $\left[\mathbf{G}_{K}\right]$ are defined on another probability space denoted by $\left(\Theta^{\prime}, \mathcal{T}^{\prime}, \mathcal{P}^{\prime}\right)$. These random matrices are such that

$$
\begin{aligned}
& {\left[\mathbf{G}_{M}\right] \in \mathbb{M}_{n}^{+}(\mathbb{R}) \text { a.s. , } E\left\{\left[\mathbf{G}_{M}\right]\right\}=\left[I_{n}\right],\left|E\left\{\log \left(\operatorname{det}\left[\mathbf{G}_{M}\right]\right)\right\}\right|<+\infty} \\
& {\left[\mathbf{G}_{K}\right] \in \mathbb{M}_{n}^{+}(\mathbb{R}) \text { a.s. }, E\left\{\left[\mathbf{G}_{K}\right]\right\}=\left[I_{n}\right],\left|E\left\{\log \left(\operatorname{det}\left[\mathbf{G}_{K}\right]\right)\right\}\right|<+\infty .}
\end{aligned}
$$

The probability density functions of random matrices $\left[\mathbf{G}_{M}\right]$ and $\left[\mathbf{G}_{K}\right]$, which are explicitly defined in [25], depend on the dispersion parameters $\delta_{M}$ and $\delta_{K}$ which have to be identified using the experimental values of the eigenfrequencies.

For any realization $\boldsymbol{X}(\theta)$ of random variable $\boldsymbol{X}$ with $\theta$ in $\Theta$ and for any independent realizations $\left[\mathbf{G}_{M}\left(\theta^{\prime}\right)\right]$ and $\left[\mathbf{G}_{K}\left(\theta^{\prime}\right)\right]$ of random matrices $\left[\mathbf{G}_{M}\right]$ and $\left[\mathbf{G}_{K}\right]$ with $\theta^{\prime}$ in $\Theta^{\prime}$, the realizations $\left[\widetilde{\boldsymbol{M}}\left(\theta^{\prime} ; \boldsymbol{X}(\theta)\right)\right]$ and $\left[\widetilde{\boldsymbol{K}}\left(\theta^{\prime} ; \boldsymbol{X}(\theta)\right)\right]$ of the random matrices $[\widetilde{\boldsymbol{M}}(\boldsymbol{X})]$ and $[\widetilde{\boldsymbol{K}}(\boldsymbol{X})]$ defined on the probability space $\left(\Theta \times \Theta^{\prime}, \mathcal{T}^{\prime} \otimes \mathcal{T}^{\prime}, \mathcal{P} \otimes \mathcal{P}^{\prime}\right)$ are written as

$$
\begin{gathered}
{\left[\widetilde{\boldsymbol{M}}\left(\theta^{\prime} ; \boldsymbol{X}(\theta)\right)\right]=\left[L_{M}(\boldsymbol{X}(\theta))\right]^{T}\left[\mathbf{G}_{M}\left(\theta^{\prime}\right)\right]\left[L_{M}(\boldsymbol{X}(\theta))\right]} \\
{\left[\widetilde{\boldsymbol{K}}\left(\theta^{\prime} ; \boldsymbol{X}(\theta)\right)\right]=\left[L_{K}(\boldsymbol{X}(\theta))\right]^{T}\left[\mathbf{G}_{K}\left(\theta^{\prime}\right)\right]\left[L_{K}(\boldsymbol{X}(\theta))\right] .}
\end{gathered}
$$

The realizations $F_{1}\left(\theta, \theta^{\prime}\right) \leq \ldots \leq F_{m}\left(\theta, \theta^{\prime}\right)$ of the random eigenfrequencies $F_{1} \leq \ldots \leq F_{m}$ are calculated by finding the solutions $\Omega\left(\theta, \theta^{\prime}\right)=2 \pi F\left(\theta, \theta^{\prime}\right)$ and $\boldsymbol{V}\left(\theta, \theta^{\prime}\right)$ of the random generalized eigenvalue problem

$$
\left[\widetilde{\boldsymbol{K}}\left(\theta^{\prime} ; \boldsymbol{X}(\theta)\right)\right] \boldsymbol{V}\left(\theta, \theta^{\prime}\right)=\Omega\left(\theta, \theta^{\prime}\right)^{2}\left[\widetilde{\boldsymbol{M}}\left(\theta^{\prime} ; \boldsymbol{X}(\theta)\right)\right] \boldsymbol{V}\left(\theta, \theta^{\prime}\right)
$$

\section{Estimation of the parameters of the probability models of un- certainties}

This section is devoted to the experimental identification of the parameters introduced in the probabilistic models of uncertainties constructed in the previous section. These parameters consist of mean value $\underline{\boldsymbol{x}}$ and dispersion parameters $\boldsymbol{\delta}_{X}, \delta_{M}$ and $\delta_{K}$. A first numerical investigation has been done with the stochastic computational model in order to analyze the sensitivity of the third eigenfrequency with respect to uncertainties. We have seen, un- 
like the first two eigenfrequencies, that (1) the third eigenfrequency cannot reasonably be fitted with experiments using only system-parameters uncertainties $\left(\boldsymbol{\delta}_{X}>0\right.$ and $\left.\delta_{M}=\delta_{K}=0\right)$ and (2) this third eigenfrequency is very sensitive to model uncertainties $\left(\boldsymbol{\delta}_{X}>0, \delta_{M}>0\right.$ and $\left.\delta_{K}>0\right)$. Consequently, only the first two eigenfrequencies will be used to estimate system-parameters uncertainties $\left(\boldsymbol{\delta}_{X}>0, \delta_{M}=0\right.$ and $\left.\delta_{K}=0\right)$. This is also the reason why the first three eigenfrequencies have not been used to simultaneously identify all the unknown parameters which are $\underline{\boldsymbol{x}}, \boldsymbol{\delta}_{X}, \delta_{M}$ and $\delta_{K}$. We have not used a unique stage to identify the unknown parameters in order to keep the spirit of the generalized probabilistic approach of uncertainties proposed in [27] for which the two types of uncertainties are separately identified. In addition, such a procedure allows the optimization cost to be reduced, above all if the dimension of the admissible set is large. Therefore, we propose two stages for identifying the unknown parameters and not a unique stage. The first one will be devoted to the identification of $\underline{\boldsymbol{x}}$ and $\boldsymbol{\delta}_{X}$ using the first two experimental eigenfrequencies (identification of the probability model of the uncertain system parameters). The second one deals with the identification of $\delta_{M}$ and $\delta_{K}$ (identification of the probability model of model uncertainties). For this second stage, several strategies are a priori possible. If the first two eigenfrequencies were not at all sensitive to model uncertainties, we would only have chosen the third experimental eigenfrequency for the identification. However, the sensitivity analysis which has been performed with respect to model uncertainties, shows that these first two eigenfrequencies are sensitive to model uncertainties. Consequently, we proposed to identify $\delta_{M}$ and $\delta_{K}$ using the first three experimental eigenfrequencies and in fixing the value of $\underline{\boldsymbol{x}}$ and $\boldsymbol{\delta}_{X}$ at the optimal values estimated in stage 1. It should be noted that another strategy could lead to another identified stochastic computational model. Nevertheless, there is no argument to decide what would be the best stochastic identified stochastic model among all the possibilities, taking into account the very small amount of available experimental data.

\subsection{Estimation of the parameters of the probability models of the uncertain system-parameters}

The parameters of the probability distribution of random variables $X_{1}, \ldots$, $X_{8}$ are (1) the vector $\underline{\boldsymbol{x}}=\left(\underline{x}_{1}, \ldots, \underline{x}_{8}\right)$ of the mean values belonging to admissible set $\mathcal{C}_{x}$ and $(2)$ the vector $\boldsymbol{\delta}_{X}=\left(\delta_{x_{1}}, \ldots, \delta_{x_{8}}\right)$ of the coefficients of variation belonging to an admissible set denoted by $\mathcal{C}_{\delta}$. Vector parameters $\underline{\boldsymbol{x}}$ and $\boldsymbol{\delta}_{X}$ must be identified using an observation of the dynamical system 
which is weakly sensitive to model uncertainties. Among all the observations of the dynamical system for which experimental measurements are available, the two lowest eigenfrequencies (fundamental eigenfrequency in each $y$-direction and $z$-direction) are the observations which are a priori the less sensitive to model uncertainties. It should be noted that only the first eigenfrequency could have been selected but due to the quasi-symmetry of the mechanical system with respect to $y$-direction and $z$-direction, the two first eigenfrequencies have been selected. The optimal values of these vector parameters are estimated using the maximum likelihood method [22],[29]. The optimal value $\underline{\boldsymbol{x}}^{\text {opt }}$ of $\underline{\boldsymbol{x}}$ and the optimal value $\boldsymbol{\delta}_{X}^{\text {opt }}$ of $\boldsymbol{\delta}_{X}$ are solution of the following optimization problem,

$$
\left(\underline{\boldsymbol{x}}^{\text {opt }}, \boldsymbol{\delta}_{X}^{\text {opt }}\right)=\arg \max _{\underline{\boldsymbol{x}} \in \mathcal{C}_{\boldsymbol{x}}, \boldsymbol{\delta}_{X} \in \mathcal{C}_{\delta}} \sum_{i=1}^{11} \log \left(p_{F_{1}, F_{2}}\left(f_{1}^{\exp , i}, f_{2}^{\text {exp }, i} ; \underline{\boldsymbol{x}}, \boldsymbol{\delta}_{X}\right)\right)
$$

in which $p_{F_{1}, F_{2}}\left(f_{1}, f_{2} ; \underline{\mathbf{x}}, \boldsymbol{\delta}_{X}\right)$ is the joint probability density function of random variables $F_{1} \leq F_{2}$ calculated using the stochastic computational model (with $\delta_{M}=\delta_{K}=0$ ) and where $f_{1}^{\exp , 1}, \ldots, f_{1}^{\exp , 11}$ and $f_{2}^{\exp , 1}, \ldots, f_{2}^{\exp , 11}$ are the 11 experimental values of the first and second eigenfrequencies defined in Table 1. The estimation of the likelihood function is performed using the Monte Carlo simulation method (see [18]) with $n_{s}=350$ simulations for which the convergence is achieved. The optimization problem is a nonconvex optimization problem on an admissible set which has a relative high dimension. Consequently, we cannot prove the existence of a unique global optimum and even if a unique global optimum existed, it cannot surely be estimated with a reasonable numerical cost. In such a problem, we can only improve the solution corresponding to the starting point and the improvement is directly proportional to the numerical cost. In this context, this optimization problem is solved using a genetic algorithm. The optimal solution is given in Table 3. The results given in Table 3 are coherent with respect to the sources of uncertainties. The dispersion on steel plate are relatively small while the dispersion on the concrete slab is larger and the dispersion of the rotation stiffness is very large, that was expected.

\subsection{Estimation of the dispersion parameters of the probability model of model uncertainties}

The dispersion parameter $\boldsymbol{\delta}_{M K}=\left(\delta_{M}, \delta_{K}\right)$ which controls the level of model uncertainties must be identified using all the observations for which 


\begin{tabular}{|c|c|c|c|}
\hline & Nominal value & Mean value & Coef. var. \\
\hline$\rho_{c s}$ & $2.4 \times 10^{3} \mathrm{~kg} / \mathrm{m}^{3}$ & $2.18 \times 10^{3} \mathrm{~kg} / \mathrm{m}^{3}$ & 0.3 \\
\hline$\lambda_{c s}$ & $2.75 \times 10^{9} \mathrm{~m}^{2} / \mathrm{N}$ & $3.30 \times 10^{9} \mathrm{~m}^{2} / \mathrm{N}$ & 0.08 \\
\hline$\mu_{c s}$ & $5.34 \times 10^{9} \mathrm{~m}^{2} / \mathrm{N}$ & $6.41 \times 10^{9} \mathrm{~m}^{2} / \mathrm{N}$ & 0.3 \\
\hline$\rho_{m p}$ & $7.8 \times 10^{3} \mathrm{~kg} / \mathrm{m}^{3}$ & $8.74 \times 10^{3} \mathrm{~kg} / \mathrm{m}^{3}$ & 0.001 \\
\hline$\lambda_{m p}$ & $1.21 \times 10^{11} \mathrm{~m}^{2} / \mathrm{N}$ & $1.07 \times 10^{11} \mathrm{~m}^{2} / \mathrm{N}$ & 0.12 \\
\hline$\mu_{m p}$ & $8.08 \times 10^{10} \mathrm{~m}^{2} / \mathrm{N}$ & $8.4 \times 10^{10} \mathrm{~m}^{2} / \mathrm{N}$ & 0.039 \\
\hline$I_{h}$ & $3.36 \times 10^{-5} \mathrm{~m}^{4}$ & $3.76 \times 10^{-5} \mathrm{~m}^{4}$ & 0.12 \\
\hline$k_{s}$ & $6.8 \times 10^{7} \mathrm{Nm} / \mathrm{rad}$ & $7.9 \times 10^{7} \mathrm{Nm} / \mathrm{rad}$ & 0.42 \\
\hline
\end{tabular}

Table 3: Optimal solution for system-parameters uncertainties identification.

experimental data are available. Consequently, the first three eigenfrequencies are used to perform the identification of $\boldsymbol{\delta}_{M K}$. Let $\mathcal{C}_{M K}$ be the admissible space of $\boldsymbol{\delta}_{M K}$. The optimal value $\boldsymbol{\delta}_{M K}^{\text {opt }}$ of $\boldsymbol{\delta}_{M K}$ is also estimated using the maximum likelihood method. As noted in Section 2, the third eigenfrequency has been measured for only 5 of the 11 motors of the family (see Table 1 ). Then, $\boldsymbol{\delta}_{M K}^{\text {opt }}$ is solution of the following optimization problem

$$
\boldsymbol{\delta}_{M K}^{\text {opt }}=\arg \max _{\delta_{M K} \in \mathcal{C}_{M K}} \mathcal{L}\left(\boldsymbol{\delta}_{M K}\right)
$$

with

$$
\begin{aligned}
\mathcal{L}\left(\boldsymbol{\delta}_{M K}\right)=\sum_{i=1}^{5} & \log \left(p_{F_{1}, F_{2}, F_{3}}\left(f_{1}^{\exp , i}, f_{2}^{\exp , i}, f_{3}^{\exp , i} ; \boldsymbol{\delta}_{M K}, \underline{\boldsymbol{x}}^{\text {opt }}, \boldsymbol{\delta}_{X}^{\text {opt }}\right)\right) \\
& +\sum_{i=6}^{11} \log \left(p_{F_{1}, F_{2}}\left(f_{1}^{\exp , i}, f_{2}^{\exp , i} ; \boldsymbol{\delta}_{M K}, \underline{\boldsymbol{x}}^{\text {opt }}, \boldsymbol{\delta}_{X}^{\text {opt }}\right)\right)
\end{aligned}
$$

in which $p_{F_{1}, F_{2}, F_{3}}\left(f_{1}, f_{2}, f_{3} ; \boldsymbol{\delta}_{M K}, \underline{\boldsymbol{x}}^{\text {opt }}, \boldsymbol{\delta}_{X}^{\text {opt }}\right)$ is the joint probability density function of random variables $F_{1} \leq F_{2} \leq F_{3}$ calculated using the stochastic computational model and where $f_{1}^{\exp , i}, f_{2}^{\exp , i}, f_{3}^{\exp , i}$ are the experimental values defined in Table 1. The estimation of the likelihood function is performed using the Monte Carlo simulation method with $n_{s}=350$ simulations for which the convergence is achieved. The graph of $\left(\delta_{M}, \delta_{K}\right) \mapsto \mathcal{L}\left(\boldsymbol{\delta}_{M K}\right)$ is plotted in Fig. 6. The maximum is reached for $\boldsymbol{\delta}_{M K}^{o p t}=(0.09,0.04)$ which corresponds to a reasonable level of model uncertainties. It should be noted 
that model uncertainties increase with the frequency in such a dynamical system. Consequently, if experimental data for higher eigenfrequencies or frequency response functions had been available, the identified level of model uncertainties would have been greater.

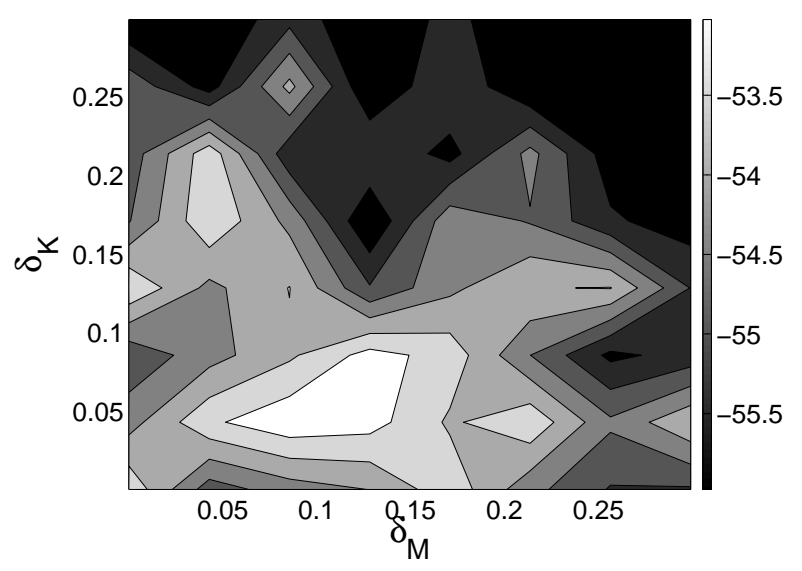

Figure 6: Graph of the likelihood function $\left(\delta_{M}, \delta_{K}\right) \mapsto \mathcal{L}\left(\boldsymbol{\delta}_{M K}\right)$.

\section{Statistics for the eigenfrequencies}

In this section, we estimate statistics for the first three eigenfrequencies using the stochastic computational model constructed in Section 4 and identified in Section 5. The statistics are estimated using the Monte carlo simulation method for two cases: (1) without taking into account model uncertainties, i.e. $\left(\underline{\boldsymbol{x}}, \boldsymbol{\delta}_{X}\right)=\left(\underline{\boldsymbol{x}}^{\text {opt }}, \boldsymbol{\delta}_{X}^{\text {opt }}\right)$ and $\boldsymbol{\delta}_{M K}=(0,0)$ and (2) taking into account model uncertainties, i.e. $\left(\underline{\boldsymbol{x}}, \boldsymbol{\delta}_{X}\right)=\left(\underline{\boldsymbol{x}}^{\text {opt }}, \boldsymbol{\delta}_{X}^{\text {opt }}\right)$ and $\boldsymbol{\delta}_{M K}=\boldsymbol{\delta}_{M K}^{\text {opt }}$. The probability density function of the first three eigenfrequencies estimated using the stochastic computational model with $\left(\underline{\boldsymbol{x}}, \boldsymbol{\delta}_{X}\right)=\left(\underline{\boldsymbol{x}}^{\text {opt }}, \boldsymbol{\delta}_{X}^{\text {opt }}\right)$ and $\boldsymbol{\delta}_{M K}=(0,0)$ are plotted in Figs. 7, 8 and 9. The probability density function of the first three eigenfrequencies estimated using the stochastic computational model with $\left(\underline{\boldsymbol{x}}, \boldsymbol{\delta}_{X}\right)=\left(\underline{\boldsymbol{x}}^{o p t}, \boldsymbol{\delta}_{X}^{\text {opt }}\right)$ and $\boldsymbol{\delta}_{M K}=\boldsymbol{\delta}_{M K}^{o p t}$ are plotted in Figs. 10, 11 and 12 . We are interested in the probability that each random eigenfrequency belongs to the $[0.92,1.08]$ critical range. The results for the two cases (without taking into account model uncertainties and taking into account model uncertainties) are respectively reported on Table 4 and Table 5 . We can remark that 


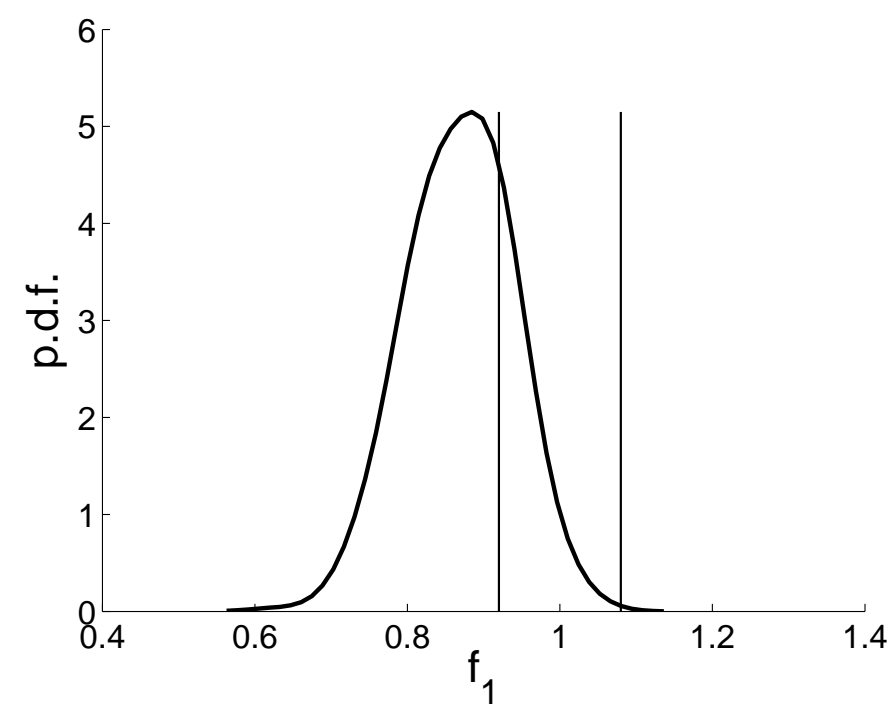

Figure 7: Probability density function of the first dimensionless eigenfrequency with $\left(\underline{\mathbf{x}}, \boldsymbol{\delta}_{X}\right)=\left(\underline{\boldsymbol{x}}^{o p t}, \boldsymbol{\delta}_{X}^{o p t}\right)$ and $\boldsymbol{\delta}_{M K}=(0,0)$. The vertical lines delimit the $[0.92,1.08]$ critical range.

\begin{tabular}{|c|c|c|c|c|}
\hline & Nominal & Mean & Coeff of var & Proba $\in[0.92,1.08]$ \\
\hline first eigenfreq. & 0.82 & 0.87 & $7.5 \%$ & 0.24 \\
\hline second eigenfreq. & 0.87 & 0.92 & $6.8 \%$ & 0.52 \\
\hline third eigenfreq. & 1.11 & 1.16 & $6.8 \%$ & 0.16 \\
\hline
\end{tabular}

Table 4: Statistics for the three first dimensionless eigenfrequencies with $\left(\underline{\boldsymbol{x}}, \boldsymbol{\delta}_{X}\right)=$ $\left(\underline{\boldsymbol{x}}^{o p t}, \boldsymbol{\delta}_{X}^{o p t}\right)$ and $\boldsymbol{\delta}_{M K}=(0,0)$.

\begin{tabular}{|c|c|c|c|c|}
\hline & Nominal & Mean & Coeff of var & Proba $\in[0.92,1.08]$ \\
\hline first eigenfreq. & 0.82 & 0.87 & $7.0 \%$ & 0.24 \\
\hline second eigenfreq. & 0.87 & 0.93 & $6.5 \%$ & 0.58 \\
\hline third eigenfreq. & 1.11 & 1.16 & $6.4 \%$ & 0.13 \\
\hline
\end{tabular}

Table 5: Statistics for the three first dimensionless eigenfrequencies with $\left(\underline{\boldsymbol{x}}, \boldsymbol{\delta}_{X}\right)=$ $\left(\underline{\boldsymbol{x}}^{o p t}, \boldsymbol{\delta}_{X}^{o p t}\right)$ and $\boldsymbol{\delta}_{M K}=\boldsymbol{\delta}_{M K}^{o p t}$. 


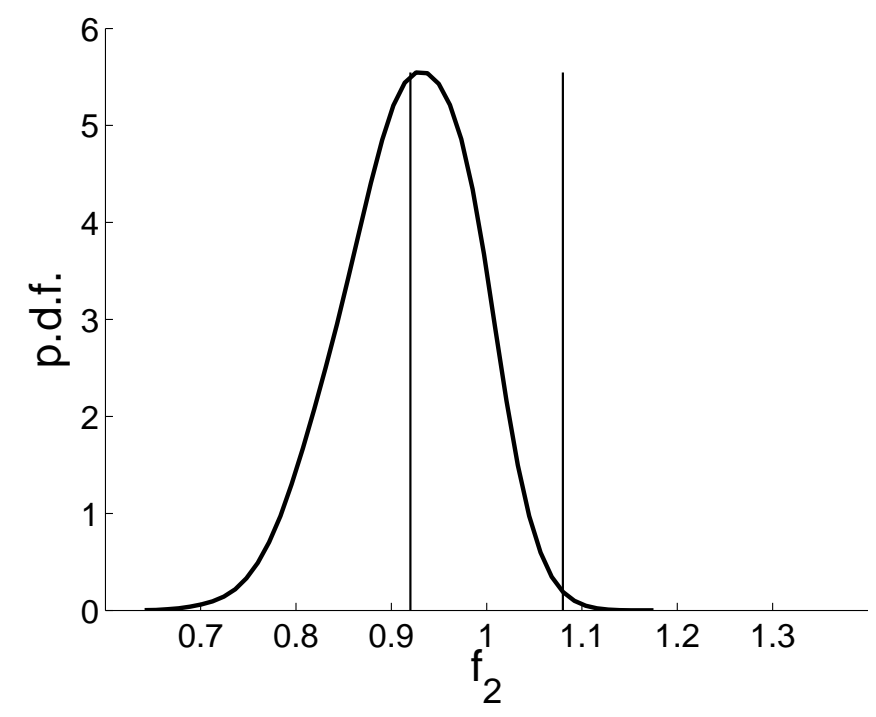

Figure 8: Probability density function of the second dimensionless eigenfrequency with $\left(\underline{\mathbf{x}}, \boldsymbol{\delta}_{X}\right)=\left(\underline{\boldsymbol{x}}^{o p t}, \boldsymbol{\delta}_{X}^{o p t}\right)$ and $\boldsymbol{\delta}_{M K}=(0,0)$. The vertical lines delimit the $[0.92,1.08]$ critical range.

model uncertainties modify the probability of being in the $[0.92,1.08]$ critical range. We can also remark that, when model uncertainties are taken into account, the coefficient of variation of the first three eigenfrequencies lightly decreases. It should be noted that, if the random observation (the eigenfrequency) was the addition of a random quantity relative to system parameter uncertainties with another random quantity relative to model uncertainties, then the variance of the random observation would be an increasing function of the variance of each random quantity. In the present case, the random observation is a nonlinear transformation of these two random quantities and consequently, the above result does not hold in the general case. The joint probability density functions $\left(f_{1}, f_{2}\right) \mapsto p_{F_{1}, F_{2}}\left(f_{1}, f_{2}\right),\left(f_{1}, f_{3}\right) \mapsto p_{F_{1}, F_{3}}\left(f_{1}, f_{3}\right)$ and $\left(f_{2}, f_{3}\right) \mapsto p_{F_{2}, F_{3}}\left(f_{2}, f_{3}\right)$ estimated using the stochastic computational model with $\left(\underline{\boldsymbol{x}}, \boldsymbol{\delta}_{X}\right)=\left(\underline{\boldsymbol{x}}^{\text {opt }}, \boldsymbol{\delta}_{X}^{\text {opt }}\right)$ and $\boldsymbol{\delta}_{M K}=(0,0)$ are plotted in Figs. 13, 14 and 15. The joint probability density functions $\left(f_{1}, f_{2}\right) \mapsto p_{F_{1}, F_{2}}\left(f_{1}, f_{2}\right)$, $\left(f_{1}, f_{3}\right) \mapsto p_{F_{1}, F_{3}}\left(f_{1}, f_{3}\right)$ and $\left(f_{2}, f_{3}\right) \mapsto p_{F_{2}, F_{3}}\left(f_{2}, f_{3}\right)$ estimated using the stochastic computational model with $\left(\underline{\boldsymbol{x}}, \boldsymbol{\delta}_{X}\right)=\left(\underline{\boldsymbol{x}}^{\text {opt }}, \boldsymbol{\delta}_{X}^{\text {opt }}\right)$ and $\boldsymbol{\delta}_{M K}=\boldsymbol{\delta}_{M K}^{\text {opt }}$ are plotted in Figs. 16, 17 and 18. We are also interested in the joint prob- 


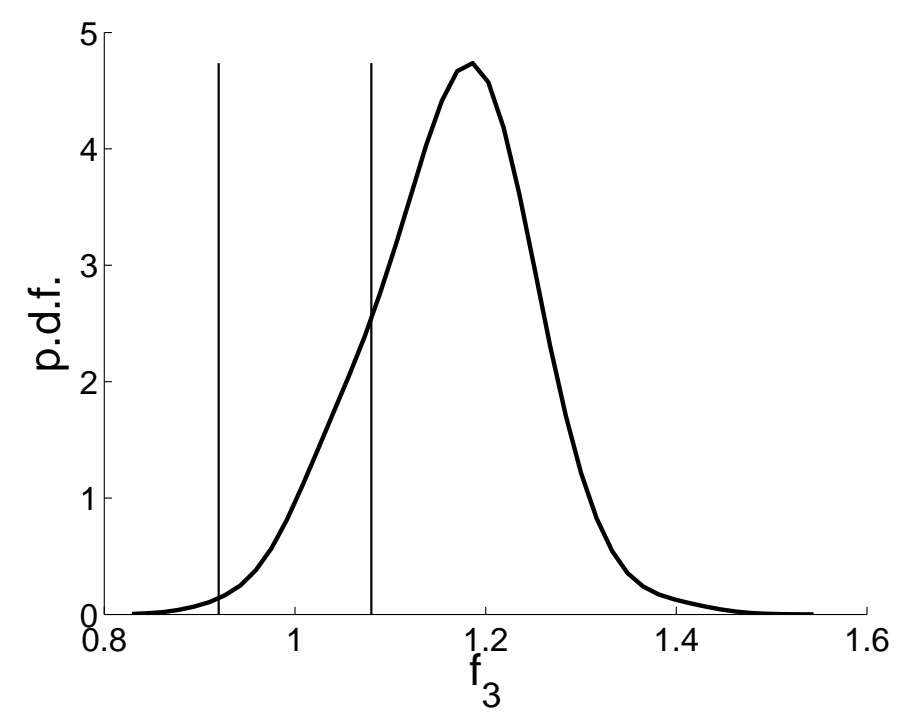

Figure 9: Probability density function of the third dimensionless eigenfrequency with $\left(\underline{\mathbf{x}}, \boldsymbol{\delta}_{X}\right)=\left(\underline{\boldsymbol{x}}^{o p t}, \boldsymbol{\delta}_{X}^{o p t}\right)$ and $\boldsymbol{\delta}_{M K}=(0,0)$. The vertical lines delimit the $[0.92,1.08]$ critical range.

ability that the first eigenfrequency belongs to the $[0.92,1.08]$ critical range. For the two cases (without taking into account model uncertainties and taking into account model uncertainties, the results are respectively reported in Table 6 and Table 7 .

\begin{tabular}{|c|c|}
\hline Event & Probability \\
\hline$\left\{F_{1} \in[0.92,1.08] \cup F_{2} \in[0.92,1.08] \cup F_{3} \in[0.92,1.08]\right\}$ & 0.65 \\
\hline$\left\{F_{1} \notin[0.92,1.08] \cap F_{2} \notin[0.92,1.08] \cap F_{3} \notin[0.92,1.08]\right\}$ & 0.35 \\
\hline$\left\{F_{1} \in[0.92,1.08] \cap F_{2} \in[0.92,1.08] \cap F_{3} \in[0.92,1.08]\right\}$ & 0.0 \\
\hline
\end{tabular}

Table 6: Statistics for the three first dimensionless eigenfrequencies with $\left(\underline{\boldsymbol{x}}, \boldsymbol{\delta}_{X}\right)=$ $\left(\underline{\boldsymbol{x}}^{\text {opt}}, \boldsymbol{\delta}_{X}^{o p t}\right)$ and $\boldsymbol{\delta}_{M K}=(0,0)$.

It can be noted that model uncertainties modify the joint probabilities of being in the $[0.92,1.08]$ critical range. Model uncertainties could be reduced by improving the nominal computational model. 


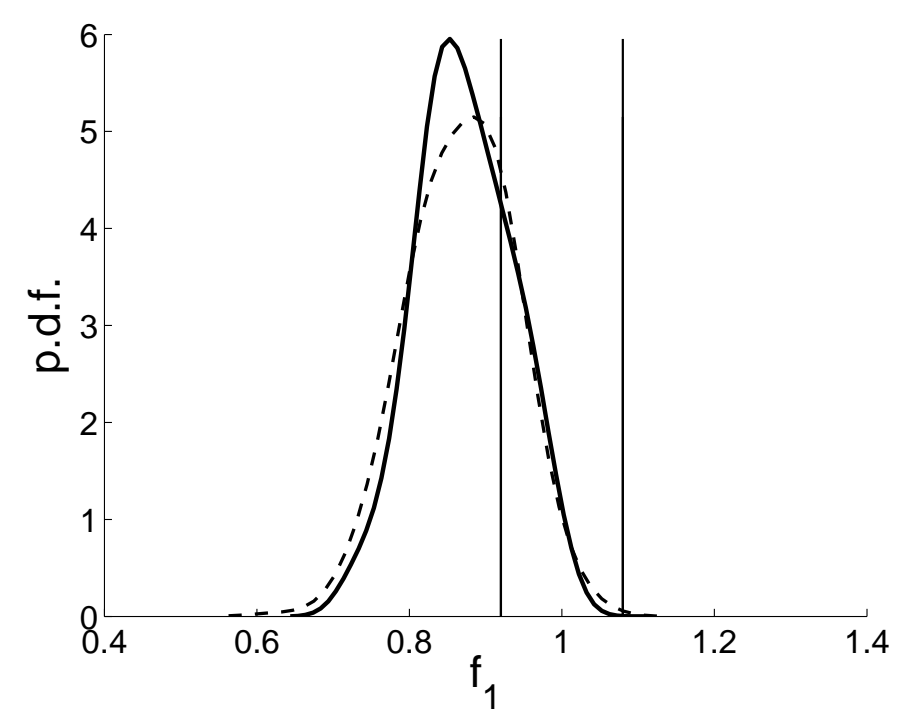

Figure 10: Probability density function of the first dimensionless eigenfrequency: with $\left(\underline{\boldsymbol{x}}, \boldsymbol{\delta}_{X}\right)=\left(\underline{\boldsymbol{x}}^{o p t}, \boldsymbol{\delta}_{X}^{o p t}\right)$ and $\boldsymbol{\delta}_{M K}=\boldsymbol{\delta}_{M K}^{o p t}$ (thick solid line), with $\left(\underline{\boldsymbol{x}}, \boldsymbol{\delta}_{X}\right)=\left(\underline{\boldsymbol{x}}^{o p t}, \boldsymbol{\delta}_{X}^{o p t}\right)$ and $\boldsymbol{\delta}_{M K}=(0,0)$ (dashed thin line). The vertical lines delimit the $[0.92,1.08]$ critical range.

\begin{tabular}{|c|c|}
\hline Event & Probability \\
\hline$\left\{F_{1} \in[0.92,1.08] \cup F_{2} \in[0.92,1.08] \cup F_{3} \in[0.92,1.08]\right\}$ & 0.70 \\
\hline$\left\{F_{1} \notin[0.92,1.08] \cap F_{2} \notin[0.92,1.08] \cap F_{3} \notin[0.92,1.08]\right\}$ & 0.30 \\
\hline$\left\{F_{1} \in[0.92,1.08] \cap F_{2} \in[0.92,1.08] \cap F_{3} \in[0.92,1.08]\right\}$ & 0.0 \\
\hline
\end{tabular}

Table 7: Statistics for the three first dimensionless eigenfrequencies with $\left(\underline{\boldsymbol{x}}, \boldsymbol{\delta}_{X}\right)=$ $\left(\underline{\boldsymbol{x}}^{o p t}, \boldsymbol{\delta}_{X}^{o p t}\right)$ and $\boldsymbol{\delta}_{M K}=\boldsymbol{\delta}_{M K}^{o p t}(0,0)$.

\section{Conclusions}

In this paper, a methodology is proposed to perform the experimental identification of an uncertain computational dynamical model representing a family of mechanical systems for which available measurements exist for the first eigenfrequencies. This methodology is applied and validated in the context of an important industrial problem related to the dynamical analysis of the Containment Spray System (CSS) motors. The stochastic computational model which is developed takes into account both system-parameters uncertainties and model uncertainties. These two types of uncertainties must 


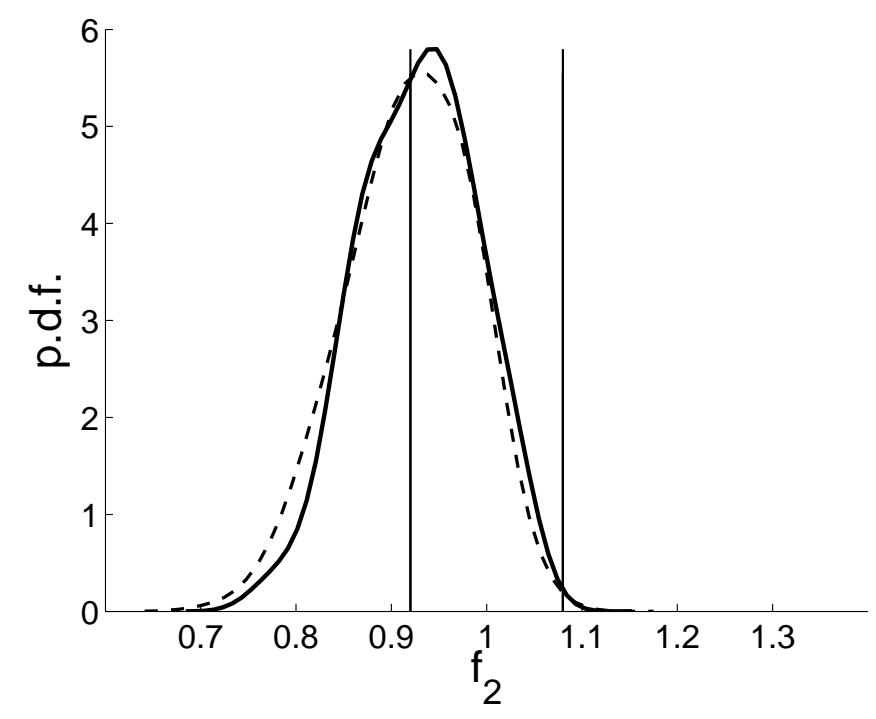

Figure 11: Probability density function of the second dimensionless eigenfrequency: with $\left(\underline{\boldsymbol{x}}, \boldsymbol{\delta}_{X}\right)=\left(\underline{\boldsymbol{x}}^{o p t}, \boldsymbol{\delta}_{X}^{o p t}\right)$ and $\boldsymbol{\delta}_{M K}=\boldsymbol{\delta}_{M K}^{o p t}$ (thick solid line), with $\left(\underline{\mathbf{x}}, \boldsymbol{\delta}_{X}\right)=\left(\underline{\boldsymbol{x}}^{o p t}, \boldsymbol{\delta}_{X}^{o p t}\right)$ and $\boldsymbol{\delta}_{M K}=(0,0)$ (dashed thin line). The vertical lines delimit the $[0.92,1.08]$ critical range.

be taken into account because only one computational model must have the capability to represent all the elements of the family of mechanical systems. The strategy consists in using a stochastic modeling which allows the two types of uncertainties to be separated and consequently, to be separately identified with measurements. For that, a generalized probabilistic approach of uncertainties recently introduced is used for the first time with experimental data. Such a method which requires available experimental data allows the identification of each type of uncertainties to be identified. The methodology which is presented is applied to the above difficult problem for which only limited experimental data are available. The available data consist of a few measurements of the first three eigenfrequencies of the dynamical system and are not sufficient to perform a complete estimation of model uncertainties induced by modeling errors. A better estimation, that is to say, a better separation between the system-parameters uncertainties and the model uncertainties, would require additional experimental data (such as experimental frequency response functions) on the dynamical responses in a frequency band larger than the third eigenfrequency. The results presented validate the approach taking into account the difficulties of the problem under 


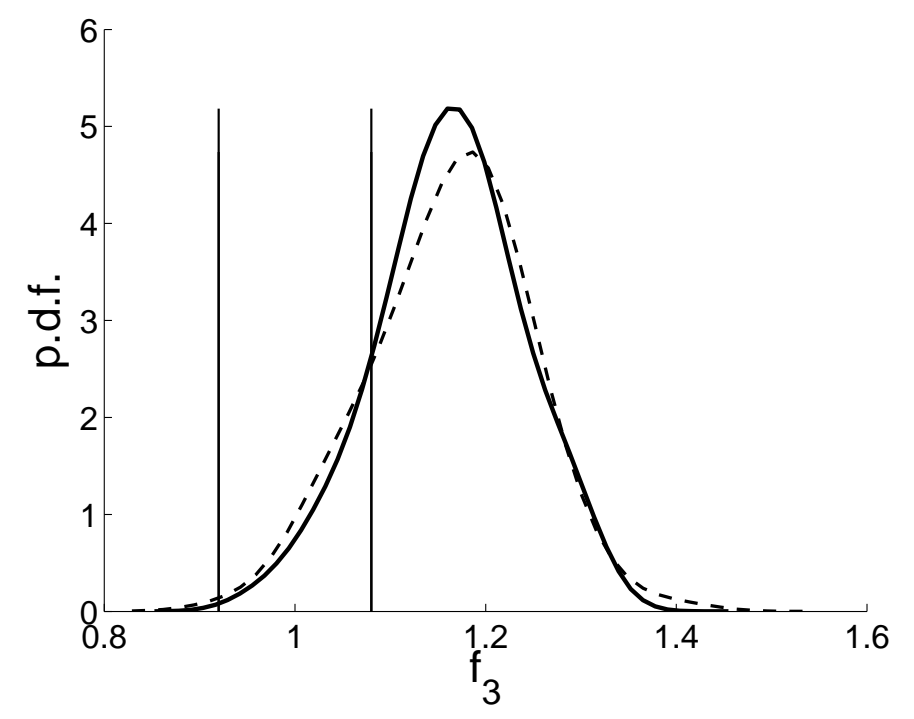

Figure 12: Probability density function of the third dimensionless eigenfrequency: with $\left(\underline{\boldsymbol{x}}, \boldsymbol{\delta}_{X}\right)=\left(\underline{\boldsymbol{x}}^{\text {opt }}, \boldsymbol{\delta}_{X}^{\text {opt }}\right)$ and $\boldsymbol{\delta}_{M K}=\boldsymbol{\delta}_{M K}^{o p t}$ (thick solid line), with $\left(\underline{\boldsymbol{x}}, \boldsymbol{\delta}_{X}\right)=\left(\underline{\boldsymbol{x}}^{\text {opt }}, \boldsymbol{\delta}_{X}^{o p t}\right)$ and $\boldsymbol{\delta}_{M K}=(0,0)$ (dashed thin line). The vertical lines delimit the $[0.92,1.08]$ critical range.

consideration. This identification has successfully been carried out despite the lack of experimental data thanks to the quality of the prior stochastic model which is constructed with Information Theory. The identified model allows a robust analysis of the dynamical system to be performed in the lowfrequency range. Finally, the methodology proposed could be used for any others dynamical systems that the presented one.

\section{Acknowledgements}

This work was supported by the French Research National Agency (ANR) (Contract TYCHE, 2010-blanc-SIMI9).

\section{References}

[1] Arnst, M., Ghanem, R., Soize, C., Identification of Bayesian posteriors for coefficients of chaos expansion, Journal of Computational Physics 229(9) (2010) 3134-3154. 


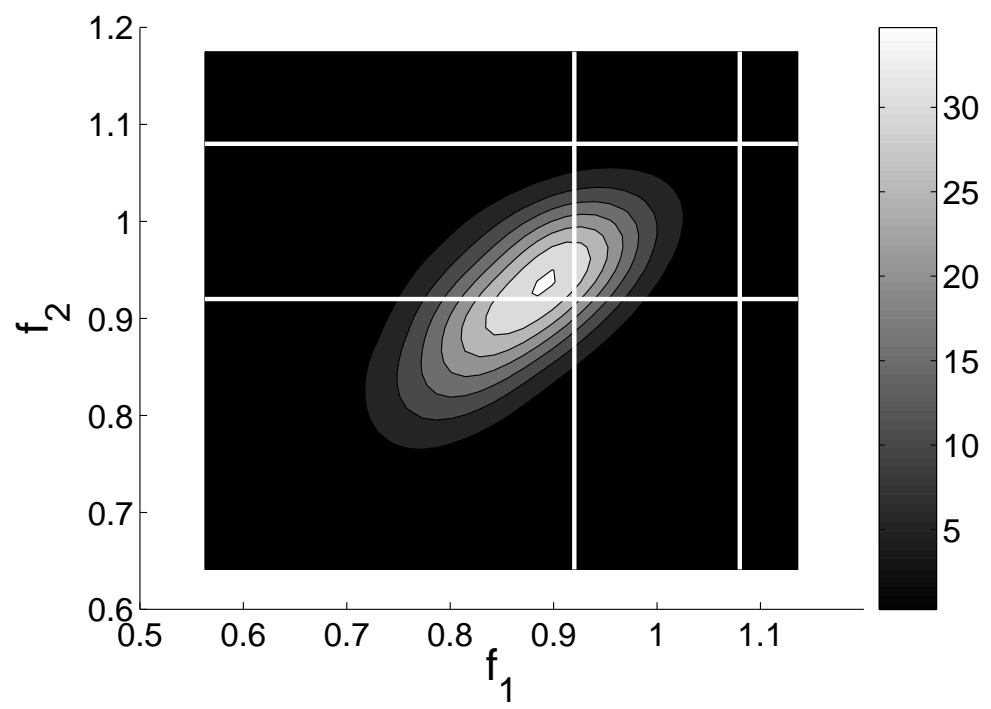

Figure 13: Joint probability density function $\left(f_{1}, f_{2}\right) \mapsto p_{F_{1}}, F_{2}\left(f_{1}, f_{2}\right)$ with $\left(\underline{\boldsymbol{x}}, \boldsymbol{\delta}_{X}\right)=$ $\left(\underline{\boldsymbol{x}}^{\text {opt }}, \boldsymbol{\delta}_{X}^{\text {opt }}\right)$ and $\boldsymbol{\delta}_{M K}=(0,0)$. The straight white lines delimit the $[0.92,1.08]$ range.

[2] Beck, J.L. and Katafygiotis, L.S., Updating models and their incertainties. I: Bayesian statistical framework, Journal of Engineering Mechanics 124(4) (1998) 455-461.

[3] Das, S., Ghanem, R. and Spall, J.C., Asymptotic sampling distribution for polynomial chaos representation from data: a maximum entropy and fisher information approach, SIAM Journal on Scientific Computing 30(5) (2007) 2207-2234.

[4] Desceliers, C., Ghanem, R. and Soize, C., Maximum likelihood estimation of stochastic chaos representations from experimental data, International Journal for Numerical Methods in Engineering 66(6) (2006) 978-1001.

[5] Ghanem, R. and Spanos, P.D, Stochastic Finite Elements: A Spectral Approach, revised edition (Dover Publications, New-York) (originally published in 1991, Springer-Verlag, New York, 2003).

[6] Ghanem, R., Masri, S., Pellissetti, M. et al, Identification and prediction of stochastic dynamical systems in a polynomial chaos basis, Computer 


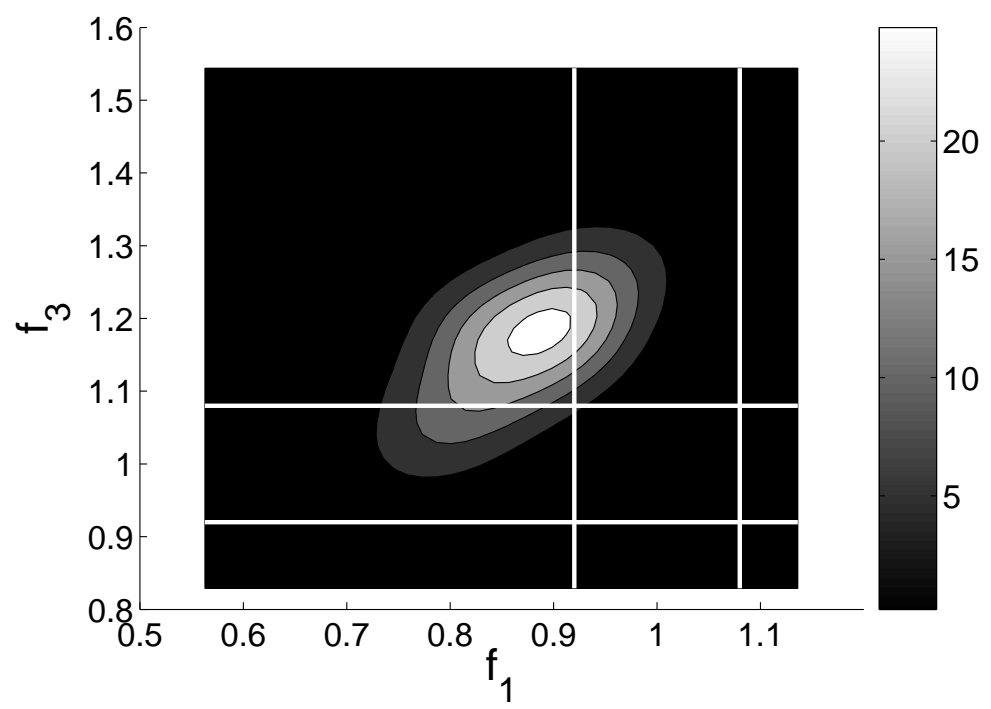

Figure 14: Joint probability density function $\left(f_{1}, f_{3}\right) \mapsto p_{F_{1}, F_{3}}\left(f_{1}, f_{3}\right)$ with $\left(\underline{\boldsymbol{x}}, \boldsymbol{\delta}_{X}\right)=$ $\left(\underline{\boldsymbol{x}}^{\text {opt }}, \boldsymbol{\delta}_{X}^{\text {opt }}\right)$ and $\boldsymbol{\delta}_{M K}=(0,0)$. The straight white lines delimit the $[0.92,1.08]$ critical range.

Methods in Applied Mechanics and Engineering 194(12-16) (2005) 16411654 .

[7] Ghanem, R. and Red-Horse J., Propagation of probabilistic uncertainty in complex physical systems using a stochastic finite element approach, Physica D 133(1-4) (1999) 137-144.

[8] Ghanem, R. and Ghosh, D., Efficient characterization of the random eigenvalue problem in a polynomial chaos decomposition, International Journal for Numerical Methods in Engineering 72(4) (2007) 486-504 (2007).

[9] Ghosh, D. and Farhat, C., Strain and stress computation in stochastic finite element methods, International Journal for Numerical Methods in Engineering 74(8) (2008) 1219-1239.

[10] Jaynes, E.T., Information theory and statistical mechanics, Physical Review 106(4) (1957) 620-630 and 108(2) (1957) 171-190. 


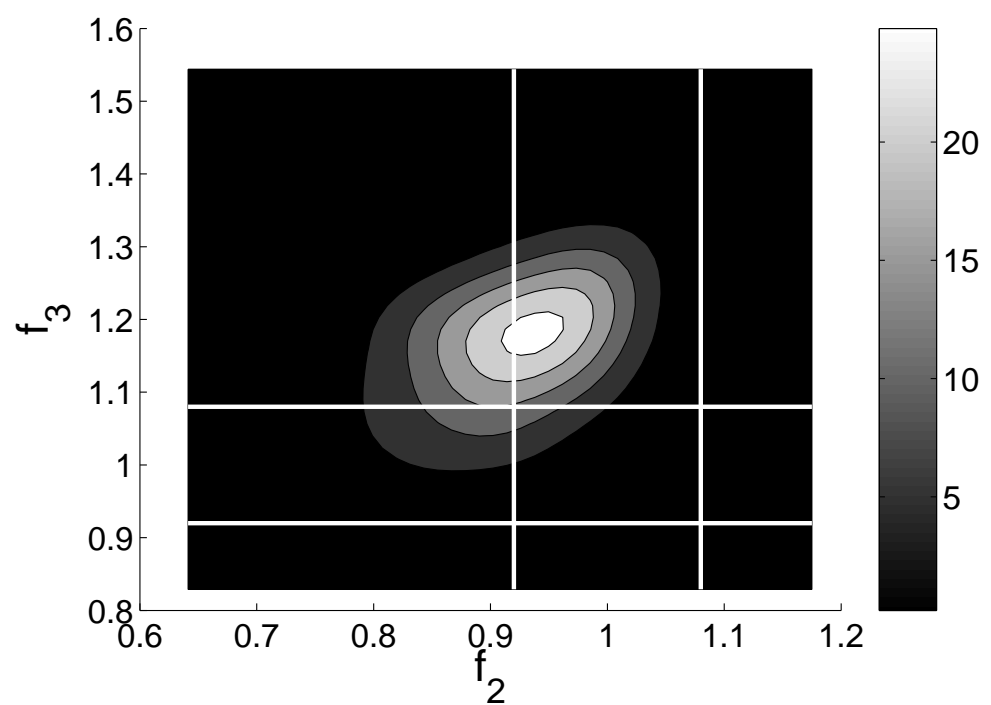

Figure 15: Joint probability density function $\left(f_{2}, f_{3}\right) \mapsto p_{F_{2}, F_{3}}\left(f_{2}, f_{3}\right)$ with $\left(\underline{\boldsymbol{x}}, \boldsymbol{\delta}_{X}\right)=$ $\left(\underline{\boldsymbol{x}}^{\text {opt }}, \boldsymbol{\delta}_{X}^{\text {opt }}\right)$ and $\boldsymbol{\delta}_{M K}=(0,0)$. The straight white lines delimit the $[0.92,1.08]$ critiacla range.

[11] Kaipo J. and Somersalo E., Statistical and Computational Inverse Problems (Springer, New York, 2005).

[12] Kapur, J.N. and Kesavan H.K. , Entropy Optimization Principles with Applications (Academic Press, San Diego, 1992).

[13] O.P. Le-Maitre, O.M. Knio, Spectral Methods for Uncerainty Quantification with Applications to Computational Fluid Dynamics, Springer, Heidelberg, 2010

[14] Le Maitre, O.P., Knio, O.M., Najm, H.N. et al., Uncertainty propagation using Wiener-Haar expansions, Journal of Computational Physics 197(1) (2004) 28-57.

[15] Mace, R.R., Worden, W., Manson, G. (editors), Uncertainty in structural dynamics, Special issue of the Journal of Sound and Vibration 288(3) (2005) 431-790.

[16] Nouy, A., Clement, A., Schoefs, F. et al, An extented stochastic finite 


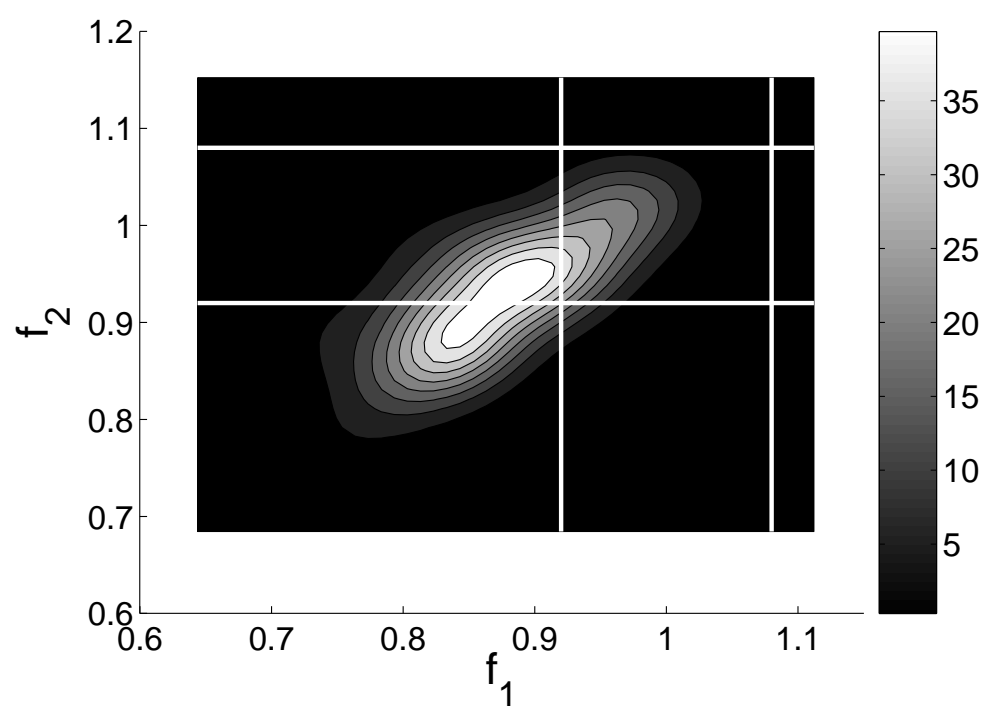

Figure 16: Joint probability density function $\left(f_{1}, f_{2}\right) \mapsto p_{F_{1}, F_{2}}\left(f_{1}, f_{2}\right)$ with $\left(\underline{\boldsymbol{x}}, \boldsymbol{\delta}_{X}\right)=$ $\left(\underline{\boldsymbol{x}}^{o p t}, \boldsymbol{\delta}_{X}^{o p t}\right)$ and $\boldsymbol{\delta}_{M K}=\boldsymbol{\delta}_{M K}^{o p t}$. The straight white lines delimit the $[0.92,1.08]$ critical range.

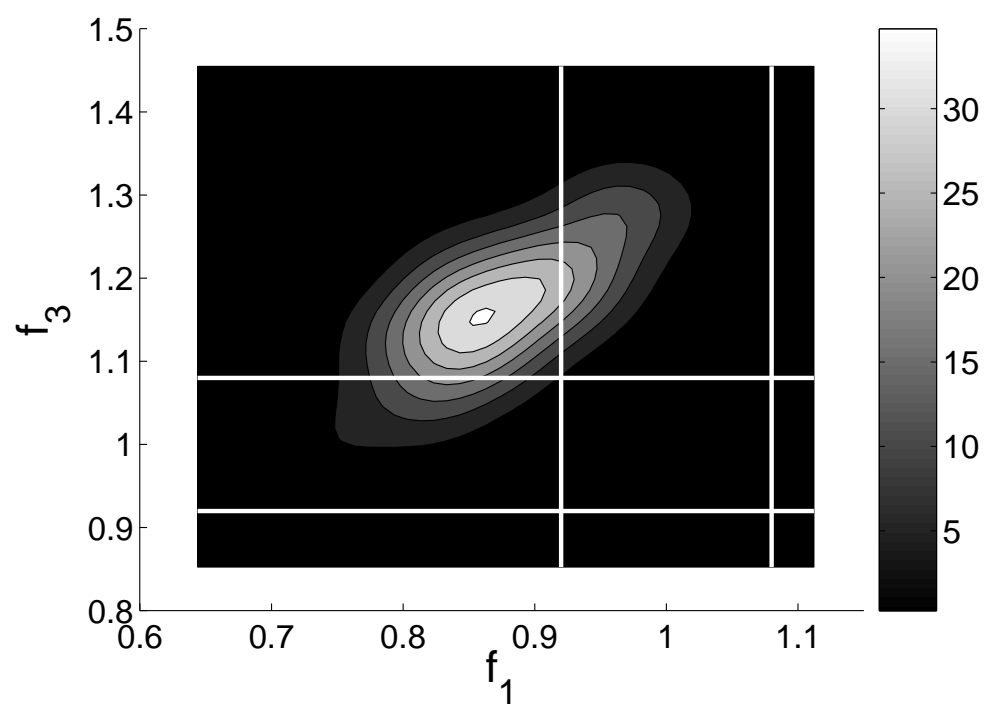

Figure 17: Joint probability density function $\left(f_{1}, f_{3}\right) \mapsto p_{F_{1}, F_{3}}\left(f_{1}, f_{3}\right)$ with $\left(\underline{\boldsymbol{x}}, \boldsymbol{\delta}_{X}\right)=$ $\left(\underline{\boldsymbol{x}}^{\text {opt }}, \boldsymbol{\delta}_{X}^{\text {opt }}\right)$ and $\boldsymbol{\delta}_{M K}=\boldsymbol{\delta}_{M K}^{\text {opt }}$. The straight white lines delimit the $[0.92,1.08]$ critical range. 


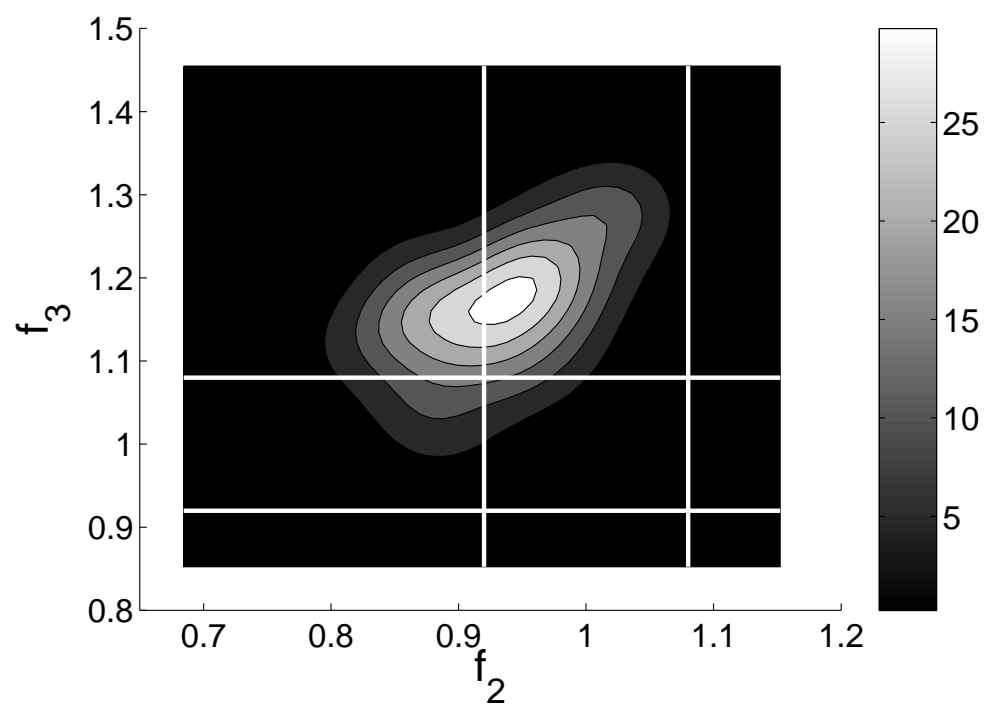

Figure 18: Joint probability density function $\left(f_{2}, f_{3}\right) \mapsto p_{F_{2}, F_{3}}\left(f_{2}, f_{3}\right)$ with $\left(\underline{\boldsymbol{x}}, \boldsymbol{\delta}_{X}\right)=$ $\left(\underline{\boldsymbol{x}}^{o p t}, \boldsymbol{\delta}_{X}^{o p t}\right)$ and $\boldsymbol{\delta}_{M K}=\boldsymbol{\delta}_{M K}^{o p t}$.The straight white lines delimit the $[0.92,1.08]$ critical range.

element method for solving stochastic partial differential equations on random domains, Computer Methods in Applied Mechanics and Engineering 197(51-52) (2008) 4663-4682.

[17] Pradlwarter, H.J., Schueller, G.I., Szekely, G.S., Random eigenvalue problems for large systems, Computers and Structures, 80 (2002) 24152424.

[18] Rubinstein, R.Y, Simulation and the Monte Carlo Method, John Wiley and Sons, 1980.

[19] Schueller, G.I., Computational methods in stochastic mechanics and reliability analysis, Computer Methods in Applied Mechanics and Engineering 194(12-16) (2005) 1251-1795.

[20] Schueller, G.I., Pradlwarter, H.J., Uncertain linear systems in dynamics: Retrospective and recent developments by stochastic approaches, Engineering Structures 31(11) (2009) 2507-2517.

[21] Schueller, G.I., Pradlwarter, H.J. Uncertainty analysis of complex struc- 
tural systems, International Journal for Numerical Methods in Engineering 80(6-7) (2009) 881-913.

[22] Serfling, R.J., Approximation Theorems of Mathematical Statistics, John Wiley \& Sons, 1980.

[23] Shannon, C.E., A mathematical theory of communication, Bell System Technology Journal 27 (1948) 379-423 and 623-659.

[24] Soize C, Ghanem R., Physical systems with random uncertainties : Chaos representation with arbitrary probability measure, SIAM Journal On Scientific Computing 26(2) (2004) 395-410.

[25] Soize, C., Random Matrix Theory for Modeling Random Uncertainties in Computational Mechanics, Comput. Methods Appl. Mech. Eng., 194(1216) (2005) 1333-1366.

[26] Soize, C., Construction of probability distributions in high dimension using the maximum entropy principle. Applications to stochastic processes, random fields and random matrices, International Journal for Numerical Methods in Engineering 76(10) (2008) 1583-1611.

[27] Soize, C., Generalized probabilistic approach of uncertainties in computational dynamics using random matrices and polynomial chaos decomposition, International Journal for Numerical Methods in Engineering, 81(8) (2010) 939-970.

[28] Soize, C., Identification of high-dimension polynomial chaos expansions with random coefficients for non-Gaussian tensor-valued random fields using partial and limited experimental data, Computer Methods in Applied Mechanics and Engineering 199(33-36) (2010) 2150-2164.

[29] Spall, J.C., Introduction to Stochastic Search and Optimization, John Wiley, 2003. 